Article

\title{
Insufficient Sleep and Poor Sleep Quality Completely Mediate the Relationship between Financial Stress and Dietary Risk among Higher Education Students
}

\author{
Chen Du ${ }^{1}\left[\right.$, Wenyan Wang ${ }^{1}$, Pao Ying Hsiao ${ }^{2}$, Mary-Jon Ludy ${ }^{3}\left[\right.$ and Robin M. Tucker ${ }^{1, *(1)}$ \\ 1 Department of Food Science and Human Nutrition, Michigan State University, East Lansing, MI 48824, USA; \\ duchen@msu.edu (C.D.); wangwe60@msu.edu (W.W.) \\ 2 Department of Food and Nutrition, Indiana University of Pennsylvania, Indiana, PA 15705, USA; \\ pyhsiao@iup.edu \\ 3 Department of Public and Allied Health, Bowling Green State University, Bowling Green, OH 43403, USA; \\ mludy@bgsu.edu \\ * Correspondence: tucker98@msu.edu; Tel.: +1-517-353-3408
}

check for

updates

Citation: Du, C.; Wang, W.; Hsiao, P.Y.; Ludy, M.-J.; Tucker, R.M. Insufficient Sleep and Poor Sleep Quality Completely Mediate the Relationship between Financial Stress and Dietary Risk among Higher Education Students. Behav. Sci. 2021, 11, 69. https://doi.org/ $10.3390 /$ bs11050069

Academic Editor: Michele Roccella

Received: 14 April 2021

Accepted: 28 April 2021

Published: 5 May 2021

Publisher's Note: MDPI stays neutral with regard to jurisdictional claims in published maps and institutional affiliations.

Copyright: (c) 2021 by the authors. Licensee MDPI, Basel, Switzerland. This article is an open access article distributed under the terms and conditions of the Creative Commons Attribution (CC BY) license (https:// creativecommons.org/licenses/by/ $4.0 /)$.

\begin{abstract}
The coronavirus disease 2019 (COVID-19) pandemic worsened financial stress for higher education students in the U.S. Financial stress is associated with poor dietary behaviors; however, factors that might influence this relationship are not well characterized. The present cross-sectional study investigated the associations between financial stress and dietary intake and dietary risk scores among higher education students (undergraduate and graduate students) in the U.S. and examined whether poor sleep quality and short sleep duration mediated the relationship between financial stress and dietary risk score. Validated tools were used to assess financial stress, sleep quality, sleep duration, dietary intake, and dietary risk. A total of 1280 students from three large U.S. universities completed the study. Results indicated that higher financial stress was associated with lower vegetable, fruit, fiber, and calcium intake, higher added sugar intake from sugar sweetened beverages, and higher dietary risk score. Further, the positive relationship between financial stress and dietary risk score was completely mediated by poor sleep quality among students who reported poor sleep quality and by short sleep duration among students who slept less than $7 \mathrm{~h}$ per night. These findings suggest that students might benefit from both financial management training and sleep education services to reduce undesirable dietary behaviors.
\end{abstract}

Keywords: sleep; sleep quality; sleep duration; diet; dietary intake; mediation analysis; COVID-19

\section{Introduction}

Financial stress, characterized as difficulty meeting financial obligations [1], is highly prevalent among higher education students (both undergraduate and graduate) in the U.S., and the recent pandemic has exacerbated financial stress in this population [2]. Financial concerns have been frequently cited by U.S. students as stressful or as reasons for contemplating leaving school [3-5]. The Spring 2019 National College Health Assessment reported more than one-third of college students stated that their finances had been traumatic or very difficult to handle during the past 12 months [6]. Unfortunately, the COVID-19 pandemic further worsened the financial situation for many students in higher education $[7,8]$. The National College Student COVID-19 Survey revealed that 30\% of students reported losing a job needed to help pay for college, $77 \%$ of students reported that the pandemic reduced their ability to earn income to support their education, and $64 \%$ stated that their need for financial aid increased due to the pandemic [9]. Based on the available evidence, an overwhelming number of higher education students in the U.S. face financial stress.

Financial stress is associated with various negative health behaviors, including poor dietary behaviors, which can lead to undesirable health outcomes [10-13]. Studies among higher education students report that greater financial difficulties are associated with 
skipping breakfast [10], frequent fast-food consumption [10], and restrained eating [13]. Additionally, previous work notes that the cost of food is one of the primary factors for young people in determining food selection [14]. For example, the cost of produce is the most frequently reported barrier preventing students from consuming fruit and vegetables [15]. Thus, greater financial stress is associated with more undesirable dietary behaviors. Further, poor dietary behaviors lead to undesirable health outcomes such as being overweight or obese [16,17], having a higher risk of suffering from chronic diseases [18-20], and having a greater likelihood of developing mental health problems [21,22]. In terms of mental health, recent research suggests adults who have been financially disadvantaged by the pandemic are experiencing higher levels of psychological distress than those who have not [23]. Based on the available evidence, the pandemic has placed increased financial stress on many students, and this increased stress is likely to also negatively affect dietary behaviors and health outcomes.

The current literature shows that financial stress associated with poor dietary behaviors, but these dietary behaviors are not well characterized among higher education students. Additionally, current investigations into the associations between financial stress and dietary behaviors focus on cost, but the cost of food is not easily modifiable. To our knowledge, investigation into other factors besides cost that could influence the relationship between financial stress and dietary behaviors has not been conducted. Therefore, the present study investigated other factors that could influence the relationships between financial stress and dietary intake and behaviors. These factors included sleep duration and quality.

Stress, including financial stress, can lead to short sleep duration and poor sleep quality [24-27], and these sleep problems can lead to poor dietary behaviors [28-31]. Thus, sleep may serve as a factor connecting financial stress and poor dietary behaviors. Several studies among university students report that higher financial stress scores or a greater number of financial strains predict lower sleep quality [24,32]. Further, reducing stress can improve both sleep duration and quality $[26,27]$. In terms of sleep and dietary intake, numerous studies note that short sleep duration and poor sleep quality increase undesirable dietary behaviors, such as: more frequent consumption of sweets [33], poor dietary quality [30,31], and increased total energy intake [29]. Given the relationships between financial stress and sleep and the relationships between sleep and dietary behaviors, sleep may serve as a mediator in these relationships.

To address the current knowledge gaps and to better understand the complex relationships between financial stress, sleep, and dietary behaviors, the present study: (1) examined the associations between financial stress and dietary intake and risky dietary behaviors, and (2) investigated whether short sleep duration and poor sleep quality mediated the relationship between financial stress and dietary risk. Based on the documented relationships between financial stress, sleep, and dietary behaviors, hypotheses included:

1) A higher financial stress score would be associated with a lower intake of healthy food groups and nutrients and a higher intake of unhealthy food groups and nutrients.

2) A higher financial stress score would be associated with a higher overall dietary risk score.

3) Poor sleep quality, but not good sleep quality, would mediate the relationship between financial stress and overall dietary risk score.

4) Short sleep duration, but not adequate sleep duration, would mediate the relationship between financial stress and overall dietary risk score.

\section{Materials and Methods}

\subsection{Study Population and Design}

The population of the present study was a sub-sample of a multi-country and multiinstitute cross-sectional study [34]. The details of the primary study, including study population and design were published previously [34]. In short, undergraduate and graduate students who were at least 18 years old and were studying at universities in China, 
Ireland, Malaysia, Taiwan, South Korea, the Netherlands, and the U.S. were recruited in the primary study. Only students studying at U.S. universities were included in the present study because the dietary questionnaires used in the present study were only validated in the U.S. population. The students in the present study were recruited from three large universities in Michigan, Ohio, and Pennsylvania. Eligible students filled out online surveys delivered through Qualtrics (SAP, Provo, Utah, USA) from April to the end of May 2020 during which time all three states involved in the study were under "shelter in place" orders. Informed consent was obtained from all subjects involved in the study.

\subsection{Measures}

\subsubsection{Demographics and Anthropometrics}

Eligible students self-reported age, gender, class status (undergraduate vs. graduate), residency status (domestic vs. international), weight and height in the surveys. Body mass index (BMI) was calculated using the self-reported weight and height information.

\subsubsection{Financial Stress}

Financial stress was assessed using the validated University Student Financial Stress Assessment (USFSA) tool, which is a 6-item questionnaire included in the 2010 Ohio Student Financial Wellness Survey (OSFWS) [35]. Questions 1 through 3 assess stress related to one's personal financial situation, such as being able to pay monthly expenses or being able to pay for school. Responses for these three questions range from 1 (strongly disagree) to 4 (strongly agree). Questions 4 through 6 assess stress related to the total amount of money owed, credit card debt, and student loan debt. Responses for these three questions range from 1 (does not apply/no debt) to 6 (extreme amount). The total score of the USFSA ranges from 6 to 30 with higher scores indicating higher financial stress. Participants were also asked whether their financial stress was greater, less, or unchanged during the COVID-19 pandemic compared to before the pandemic.

\subsubsection{Perceived Stress}

Perceived stress was assessed using the validated Perceived Stress Scale-10 (PSS-10) [36] The PSS-10 questionnaire included 10 items, and responses for each item range from 0 (never) to 4 (very often). Example questions are "In the last month, how often have you been upset because of something that happened unexpectedly?", and "In the last month, how often have you found that you could not cope with all the things that you had to do?" The total score of the PSS-10 ranges from 0 to 40 , with higher scores indicating more perceived stress.

\subsubsection{Sleep Quality and Duration}

Subjective sleep quality was assessed using the validated Pittsburgh Sleep Quality Index (PSQI) [37-41]. The PSQI consists of 10 main questions. The first four questions assess sleeping habits and duration; the other questions assess the degree of sleep problems. The PSQI scores range from 0 to 21, with higher scores indicating poorer sleep quality. In addition, participants were classified as poor sleepers if they reported a PSQI score of $\geq 5$ [41]. Sleep duration on weekdays and weekends were collected by asking participants how many hours they usually sleep during the weekdays and weekends. Average sleep duration was calculated using a weighted method (((weekday sleep duration $\times 5)$ $+($ weekend sleep duration $\times 2)) / 7$ ). Participants were classified as having short sleep duration if they reported a sleep duration $<7 \mathrm{~h}$ per sleep duration guidelines [42].

\subsubsection{Dietary Intake}

Dietary intake of specific food groups and nutrients was assessed using the Dietary Screener Questionnaire (DSQ) from the National Health and Nutrition Examination Survey (NHANES) 2009 to 2010 [43]. Dietary intake data were converted from intake frequencies and processed per the DSQ Data Processing and Scoring protocol [44]. The DSQ provides estimations of predicted intake of dairy (cup equivalents per day), whole grains 
(ounce equivalents per day), vegetables including legumes and excluding French fries (cup equivalents per day), fruit (cup equivalents per day), fiber (grams per day), calcium (milligrams per day), predicted intake of total added sugars (teaspoons equivalents per day), and predicted intake of added sugars from sugar-sweetened beverages (SSB, teaspoons equivalents per day) for males and females. The prediction equations used per protocol only calculate intake for males and females; thus, students who identified as "other" were not included in the analysis. The DSQ also measures frequency of processed meat and red meat consumption. Computation of consumption frequency does not require prediction equations; therefore, frequency of processed meat and red meat consumption was computed for all gender categories.

\subsubsection{Dietary Risk}

Dietary risk score was assessed using a validated eight-item simplified food frequency questionnaire, Starting the Conversation (STC) [45]. The STC survey measures eating frequencies of fast foods, fruit, vegetables, soda and sweet tea, high quality proteins, chips and crackers, dessert, and solid fat. A sample question from the STC is "How many times a week did you eat fast food meals or snacks?" Answers for eating frequencies of low nutritional quality foods range from 0 (less than 1 time) to 2 ( 4 or more times) while answers for eating frequencies of high nutritional quality foods range from 0 ( 5 or more) to 2 ( 2 or less). Summing all answers provides a global score of dietary risk behaviors ranging from 0 to 16 , with higher scores indicating more frequent engagement in dietary behaviors that are considered unhealthy.

\subsubsection{Physical Activity}

Physical activity was measured using the validated International Physical Activity Questionnaire (IPAQ) long form [46]. Participants reported walking, moderate, and vigorous activities in terms of frequency and duration for five domains of activity including occupation-related, transportation, housework, walking, and recreation. Activities for each domain were calculated per the IPAQ long form protocol [47] and were reported using metabolic equivalents (METs) minutes per week. The total METs minutes per week were the sum of the activities for each domain. Physical activity was assessed in order to determine if it should be considered a covariate in the analyses.

\subsubsection{Mediation Models}

To test whether poor sleep quality and short sleep duration mediated the relationship between financial stress and dietary risk scores, two models were built (Figure 1). Participants who had a PSQI score $\geq 5$ were included in mediation model 1, and participants who reported sleeping $<7 \mathrm{~h}$ were included in mediation model 2 . To test whether sleep quality and duration also mediate the relationship between financial stress and dietary risk scores among "good" sleepers, the same analyses were run among students who reported good sleep quality (PSQI < 5) (model 3) and who reported meeting the minimum recommended sleep duration of $7 \mathrm{~h}$ per day (model 4 ).

The dietary risk score (STC score) presents a global score for unhealthy dietary behaviors; therefore, they were used in the mediation models. Dietary intake was not examined in the models because there was no global score that could be used to represent total intake. 


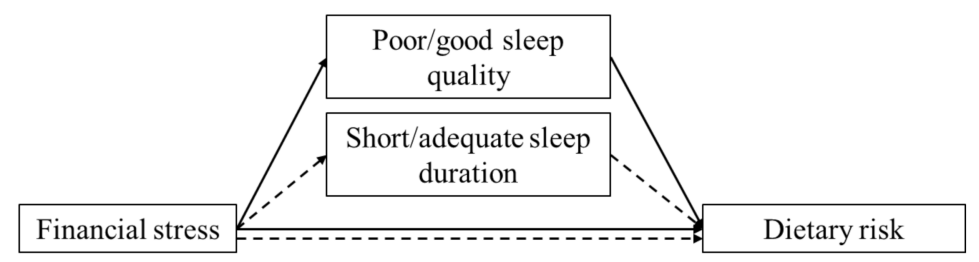

Figure 1. Proposed mediation models. The effects of financial stress on dietary risk scores through the mediation of sleep quality among poor sleepers (mediation model 1, solid line) or good sleepers (mediation model 3, solid line). The effects of financial stress on dietary risk scores through the mediation of sleep duration among students who reported short sleep duration (mediation model 2, dashed line) or students who reported adequate sleep duration (mediation model 4 , dashed line).

\subsection{Statistical Analyses}

Descriptive statistics were performed and were presented as means \pm standard deviations (SD) for continuous variables and percentages (\%) for categorical variables. Bivariate correlation analyses were conducted using Pearson correlation tests or one-way ANOVA. The purposes of the bivariate correlation analyses were to assess the relationships between the independent variable (financial stress) and covariates (age, gender, graduate status, residency status, BMI, sleep quality, sleep duration, physical activity, and perceived stress) and between the dependent variables (dietary intakes and the overall dietary risk score) and covariates. False discovery rate adjustment was performed; specifically, Benjamini-Hochberg adjusted $p$ values were used to evaluate significance for the bivariate analyses for all continuous variables [48].

Multivariate regression analyses were conducted to assess the relationships between financial stress score and dietary intake and between financial stress score and dietary risk score. Statistical assumptions of multivariate regression analyses were tested, including normality, linearity, multicollinearity, and homoscedasticity. The sample data met all assumptions. Covariates adjusted in the models were evaluated conceptually and empirically $[49,50]$. Model A of the multivariate regression analysis represents the unadjusted model. Age, gender, and residency status were adjusted in model B, and model C further adjusted for perceived stress in addition to the variables adjusted in model B.

Mediation analyses were conducted to examine whether sleep quality and sleep duration mediated the relationship between financial stress and dietary risk scores. The mediation analyses were carried out in students who reported poor sleep quality, good sleep quality, short sleep duration, and adequate sleep duration separately. This approach allows for the determination of whether these variables individually mediate the relationship between financial stress and dietary risk scores and whether the mediating relationships are only present among students who reported poor sleep quality or short sleep duration. Power analysis was based on 20 participants per construct in each model; therefore, a minimum of 60 participants were needed in each model [51]. The analyses were conducted using the SPSS PROCESS Macro, and Model 4 was chosen for the analyses [52]. Age, gender, residency status, and perceived stress were adjusted in all four models. The number of bootstraps performed for bias corrected bootstrap confidence intervals was set at 10,000. To interpret the results of the mediation analyses, the indirect effect of an independent variable on a dependent variable through the mediator (bootstrap results) must be significant in order to show a significant mediation effect. Additionally, the singular direct effects of an independent variable on a dependent variable, an independent variable on a mediating variable, or a mediating variable on a dependent variable do not need to be significant in order for the mediation effect to be significant [52,53].

The DSQ data were prepared using SAS 9.4 (SAS Institute, Cary, NC, USA) and all the analyses were performed in SPSS version 26 (IBM Corporation, Armonk, NY, USA). The threshold for statistical significance was set at $p<0.05$. 


\section{Results}

\subsection{Demographic and Anthropometric Information}

A total of 1433 students initiated the survey; 1280 students completed the survey and were included in the analyses (Table 1). The majority of the students were female, undergraduate, and domestic students. The average BMI of the students was classified as overweight. The majority of the students reported poor sleep quality while most of the students reported meeting the minimum sleep recommendation of $7 \mathrm{~h}$ per night.

Table 1. Demographic, anthropometric, and sleep characteristics.

\begin{tabular}{|c|c|c|}
\hline & \multicolumn{2}{|c|}{$\begin{array}{l}\text { U.S. Higher Education Students } \\
\qquad N=1280\end{array}$} \\
\hline & $\mathbf{N}(\%)$ & $($ Mean \pm SD) \\
\hline \multicolumn{3}{|l|}{ Gender } \\
\hline Male & $308(24)$ & - \\
\hline Female & $935(73)$ & - \\
\hline Other & $37(3)$ & - \\
\hline \multicolumn{3}{|c|}{ Graduate status } \\
\hline Undergraduate students & $951(74)$ & - \\
\hline Graduate students & $329(26)$ & - \\
\hline \multicolumn{3}{|l|}{ Residency status } \\
\hline Domestic students & $1113(87)$ & - \\
\hline International students & $167(13)$ & - \\
\hline Age $(y)$ & 1266 & $22.5 \pm 4.8$ \\
\hline $\mathrm{BMI}\left(\mathrm{kg} / \mathrm{m}^{2}\right)$ & 1273 & $25.9 \pm 6.2$ \\
\hline \multicolumn{3}{|l|}{ Sleep quality } \\
\hline Poor sleeper & $996(78)$ & $8.6 \pm 3.0$ \\
\hline Good sleeper & $284(22)$ & $3.0 \pm 1.0$ \\
\hline \multicolumn{3}{|l|}{ Sleep duration } \\
\hline $\begin{array}{l}\text { Did not meet } \\
\text { recommendations }\end{array}$ & $310(24)$ & $6.0 \pm 0.7$ \\
\hline Met recommendations & $970(76)$ & $8.2 \pm 0.9$ \\
\hline
\end{tabular}

Demographic, anthropometric, and sleep characteristics of the study sample. SD = Standard deviation. Poor sleepers were classified as reporting a PSQI score $\geq 5$. "Did not meet sleep recommendations" refers to students who reported nightly sleep duration $<7 \mathrm{~h}$. Missing Data: age $(\mathrm{N}=1)$; $\mathrm{BMI}=$ body mass index $(\mathrm{N}=7)$; gender $(\mathrm{N}=0)$; graduate status $(\mathrm{N}=0)$; residency status $(\mathrm{N}=0)$.

\subsection{Associations between Financial Stress and Demographic and Health Characteristics}

The majority of the students (52.8\%) reported having greater financial stress during the COVID-19 pandemic compared to before, $8.8 \%$ of students reported less financial stress, and $38.4 \%$ of students reported no change in financial stress. Greater financial stress was associated with older age, and female students reported greater financial stress compared to male students (Table 2). Additionally, domestic students reported greater financial stress compared to international students. In terms of health characteristics, greater financial stress was associated with higher BMI, higher perceived stress, lower sleep quality, and shorter sleep duration. 
Table 2. Financial stress and its associations with demographic and health characteristics.

\begin{tabular}{|c|c|c|}
\hline Continuous Measures & Mean \pm SD & Correlation Coefficient with Financial Stress \\
\hline Financial stress (USFSA score) & $17.4 \pm 5.9$ & - \\
\hline Age (years) & $22.5 \pm 4.8$ & $0.067 *$ \\
\hline $\mathrm{BMI}\left(\mathrm{kg} / \mathrm{m}^{2}\right)$ & $25.9 \pm 6.2$ & $0.216^{* * *}$ \\
\hline Perceived stress (PSS-10 score) & $21.7 \pm 7.1$ & $0.341^{* * *}$ \\
\hline Sleep quality (PSQI score) & $7.4 \pm 3.6$ & $0.325^{* * *}$ \\
\hline Sleep duration $(\mathrm{h})$ & $7.6 \pm 1.3$ & $-0.199 * * *$ \\
\hline $\begin{array}{l}\text { Physical activity level } \\
\text { (METs minutes per week) }\end{array}$ & $3330.5 \pm 4056.3$ & 0.034 \\
\hline Categorical Measures & $\begin{array}{c}\text { Financial Stress } \\
\text { Mean } \pm \text { SD }\end{array}$ & $p$ Value \\
\hline \multicolumn{3}{|l|}{ Gender } \\
\hline Male & $16.5 \pm 5.9^{\mathrm{a}}$ & 0.006 \\
\hline Female & $17.6 \pm 5.8^{b}$ & \\
\hline Other & $18.9 \pm 5.5^{\mathrm{a}, \mathrm{b}}$ & \\
\hline \multicolumn{3}{|l|}{ Graduate status } \\
\hline Undergraduate students & $17.5 \pm 5.9$ & 0.079 \\
\hline Graduate students & $16.9 \pm 5.7$ & \\
\hline Residency status & & \\
\hline Domestic students & $17.6 \pm 5.9$ & 0.001 \\
\hline International students & $16.0 \pm 5.2$ & \\
\hline
\end{tabular}

Financial stress as measured by the USFSA according to gender, degree, and residency status. Note that sleep quality was measured using the PSQI, and higher PSQI scores indicate lower sleep quality. ${ }^{*} p<0.05 ;{ }^{* * *} p<0.001$. Benjamini-Hochberg adjusted $p$ values were used for determinations of significance. Means with different superscripts are significantly different, $p<0.05$. SD $=$ Standard deviation. Missing Data: age $(\mathrm{N}=1)$; BMI $(\mathrm{N}=7)$; gender $(\mathrm{N}=0)$; graduate status $(\mathrm{N}=0)$; residency status $(\mathrm{N}=0)$; perceived stress $(\mathrm{N}=0)$; sleep quality $(\mathrm{N}=8)$; sleep duration $(\mathrm{N}=9)$; physical activity level $(\mathrm{N}=115)$.

\subsection{Associations between Demographic and Health Characteristics and Dietary Intakes and Behaviors}

Table 3 lists the Pearson product moment correlation values for demographic, perceived stress, sleep, and physical activity measures. Age and BMI were associated with dietary intakes and dietary risk score. Older age was associated with higher intake of whole grains, vegetables, fiber, total added sugar, and added sugar from SSB. In addition, older age was also associated with a lower dietary risk score. Higher BMI was associated with lower intake of whole grains, fruit, and fiber but with higher intake of red meat, processed meat, total added sugar, and added sugar from SSB. Higher BMI was also associated with a higher dietary risk score.

Higher perceived stress was associated with lower intake of dairy, whole grains, vegetables, fruit, red meat, fiber, and calcium but higher intake of total added sugar and added sugar from SSB. Further, higher perceived stress was associated with a higher dietary risk score.

Differences between the associations of sleep quality and duration with dietary intakes and dietary risk score were observed. Poorer sleep quality was associated with lower intake of whole grains, vegetables, fruit, fiber, calcium, and higher intake of total added sugars and added sugar from SSB. In addition, poorer sleep quality was associated with a higher dietary risk score. Shorter sleep duration was only associated with higher intake of added sugar from SSB.

Higher physical activity level was associated with higher intake of vegetables, fruit, fiber, and calcium. Furthermore, a higher level of physical activity was associated with a lower dietary risk score. 
Table 3. Dietary intake, dietary risk score, and their associations with demographic and health characteristics of U.S. higher education students (continuous variables).

\begin{tabular}{|c|c|c|c|c|c|c|c|c|c|c|c|}
\hline \multirow[b]{2}{*}{$\begin{array}{l}\text { Continuous } \\
\text { Measures }\end{array}$} & \multirow[b]{2}{*}{$\begin{array}{l}\text { Dairy } \\
\text { (cups/d) }\end{array}$} & \multirow[b]{2}{*}{$\begin{array}{l}\text { Whole } \\
\text { Grains } \\
\text { (oz/d) }\end{array}$} & \multirow[b]{2}{*}{$\begin{array}{l}\text { Vegetables } \\
\text { (cups/d) }\end{array}$} & \multirow[b]{2}{*}{$\begin{array}{c}\text { Fruit } \\
\text { (cups/d) }\end{array}$} & \multirow[b]{2}{*}{$\begin{array}{l}\text { Red Meat } \\
\text { (times/d) }\end{array}$} & \multicolumn{2}{|c|}{ Outcome Measures } & \multirow[b]{2}{*}{$\begin{array}{l}\text { Calcium } \\
\text { (mg/d) }\end{array}$} & \multirow[b]{2}{*}{$\begin{array}{l}\text { Total Added } \\
\text { Sugar(tsp/d) }\end{array}$} & \multirow[b]{2}{*}{$\begin{array}{l}\text { Added Sugar } \\
\text { from SSB } \\
\text { (tsp/d) }\end{array}$} & \multirow[b]{2}{*}{$\begin{array}{l}\text { Dietary } \\
\text { Risk Score }\end{array}$} \\
\hline & & & & & & $\begin{array}{c}\text { Processed } \\
\text { Meat } \\
\text { (times/d) }\end{array}$ & $\begin{array}{l}\text { Fiber } \\
\text { (g/d) }\end{array}$ & & & & \\
\hline Mean \pm SD & $1.6 \pm 0.6$ & $0.7 \pm 0.3$ & $1.3 \pm 0.3$ & $0.9 \pm 0.4$ & $0.3 \pm 0.3$ & $0.2 \pm 0.2$ & $15.6 \pm 3.2$ & $968.8 \pm 207.9$ & $16.3 \pm 6.0$ & $6.6 \pm 4.1$ & $8.2 \pm 2.7$ \\
\hline Age (y) & 0.018 & $0.078 * *$ & $0.233 * *$ & 0.014 & -0.021 & -0.029 & $0.148^{* * *}$ & 0.022 & $0.074 *$ & $0.071 *$ & $-0.062 *$ \\
\hline $\mathrm{BMI}\left(\mathrm{kg} / \mathrm{m}^{2}\right)$ & 0.035 & $-0.070 *$ & -0.059 & $-0.100 * * *$ & 0.067 * & $0.077^{* *}$ & $-0.125^{* * *}$ & -0.003 & $0.066^{* *}$ & $0.077^{* *}$ & $0.134^{* * *}$ \\
\hline $\begin{array}{l}\text { Perceived stress } \\
\text { (PSS-10 score) }\end{array}$ & $-0.064 *$ & $-0.079 * *$ & $-0.210^{* * *}$ & $-0.115^{* * *}$ & $-0.105^{* * *}$ & 0.004 & $-0.224^{* * *}$ & $-0.151^{* * *}$ & 0.043 & 0.020 & $0.183^{* * *}$ \\
\hline $\begin{array}{l}\text { Sleep quality } \\
\text { (PSQI score) }\end{array}$ & -0.015 & $-0.085^{* *}$ & $-0.112^{* * *}$ & $-0.111^{* * *}$ & -0.050 & 0.009 & $-0.192^{* * *}$ & $-0.092^{* *}$ & $0.083^{* *}$ & $0.103^{* * *}$ & $0.164^{* * *}$ \\
\hline Sleep duration (h) & -0.018 & 0.008 & -0.024 & 0.018 & -0.041 & -0.052 & 0.024 & -0.005 & -0.055 & $-0.099 * *$ & 0.015 \\
\hline $\begin{array}{l}\text { Physical activity } \\
\text { level (METs } \\
\text { minutes per week) }\end{array}$ & 0.033 & 0.067 & $0.121^{* * *}$ & $0.097 *$ & 0.036 & 0.056 & $0.110^{* * *}$ & $0.085 * *$ & -0.042 & -0.011 & $-0.133^{* * *}$ \\
\hline
\end{tabular}

Pearson correlation coefficients for the relationships between dietary intake, measured by DSQ, and measured variables. Note: Numbers listed in tables represent correlation coefficient. ${ }^{*} p<0.05 ;{ }^{* *} p<0.01 ;{ }^{* * *} p<0.001 ; p<0.05$ was used to determine significance and BenjaminiHochberg adjusted $\mathrm{p}$ values were used for excluding possible false positive results. All but the associations between physical activity level and whole grains remained significant after the Benjamini-Hochberg false discovery adjustment. SD = Standard deviation. Missing data: age $(\mathrm{N}=1)$; $\mathrm{BMI}(\mathrm{N}=7)$; gender $(\mathrm{N}=0)$; graduate status $(\mathrm{N}=0)$; residency status $(\mathrm{N}=0)$; perceived stress $(\mathrm{N}=0)$; sleep quality $(\mathrm{N}=8)$; sleep duration $(\mathrm{N}=9)$; physical activity level $(\mathrm{N}=115)$; dairy $(\mathrm{N}=41)$; whole grains $(\mathrm{N}=37)$; vegetables $(\mathrm{N}=37)$; fruit $(\mathrm{N}=37)$; red meat $(\mathrm{N}=4)$; processed meat $(\mathrm{N}=1276)$; fiber $(\mathrm{N}=37)$; calcium $(\mathrm{N}=37)$; total added sugar $(\mathrm{N}=40)$; total added sugar from SSB $(\mathrm{N}=52)$; dietary behaviors $(\mathrm{N}=0)$.

Female students reported lower intake of most foods except whole grains compared to male students, but the dietary risk scores measured using the STC did not differ between female and male students (Table 4). Compared to graduate students, undergraduate students consumed lower amounts of vegetables, fruits, and fiber, and reported higher dietary risk scores. Compared to international students, domestic students reported lower intake of vegetables, red meat, and fiber, and higher consumption of processed meat. Additionally, higher dietary risk scores were observed among domestic compared to international students.

Table 4. Dietary intake, dietary risk score, and their associations with demographic and health characteristics of U.S. higher education students (categorical variables).

\begin{tabular}{|c|c|c|c|c|c|c|c|c|c|c|c|}
\hline $\begin{array}{c}\text { Categorical } \\
\text { Measures }\end{array}$ & $\begin{array}{l}\text { Dairy } \\
\text { (cups/d) }\end{array}$ & $\begin{array}{l}\text { Whole } \\
\text { Grains } \\
\text { (oz/d) }\end{array}$ & $\begin{array}{l}\text { Vegetables } \\
\text { (cups/d) }\end{array}$ & $\begin{array}{c}\text { Fruit } \\
\text { (cups/d) }\end{array}$ & $\begin{array}{l}\text { Red Meat } \\
\text { (times/d) }\end{array}$ & $\begin{array}{c}\text { Processed } \\
\text { Meat } \\
\text { (times/d) }\end{array}$ & $\begin{array}{l}\text { Fiber } \\
\text { (g/d) }\end{array}$ & $\begin{array}{l}\text { Calcium } \\
\text { (mg/d) }\end{array}$ & $\begin{array}{l}\text { Total Added } \\
\text { Sugar(tsp/d) }\end{array}$ & $\begin{array}{l}\text { Added Sugar } \\
\text { from SSB } \\
\text { (tsp/d) }\end{array}$ & $\begin{array}{c}\text { Dietary } \\
\text { Risk } \\
\text { Score }\end{array}$ \\
\hline & $\mathrm{M} \pm \mathrm{SD}$ & $\mathrm{M} \pm \mathrm{SD}$ & $\mathrm{M} \pm \mathrm{SD}$ & $\mathrm{M} \pm \mathrm{SD}$ & $\mathrm{M} \pm \mathrm{SD}$ & $\mathrm{M} \pm \mathrm{SD}$ & $\mathrm{M} \pm \mathrm{SD}$ & $\mathrm{M} \pm \mathrm{SD}$ & $\mathrm{M} \pm \mathrm{SD}$ & $\mathrm{M} \pm \mathrm{SD}$ & $\mathrm{M} \pm \mathrm{SD}$ \\
\hline \multicolumn{12}{|l|}{ Gender } \\
\hline Male & $2.0 \pm 0.7$ & $0.7 \pm 0.3$ & $1.5 \pm 0.4$ & $1.0 \pm 0.5$ & $0.4 \pm 0.4$ & $0.3 \pm 0.3$ & $17.8 \pm 3.5$ & $1176.8 \pm 249.6$ & $19.3 \pm 7.8$ & $8.4 \pm 5.1$ & $8.0 \pm 2.9$ \\
\hline Female & $1.5 \pm 0.5$ & $0.7 \pm 0.3$ & $1.3 \pm 0.3$ & $0.9 \pm 0.4$ & $0.3 \pm 0.3$ & $0.2 \pm 0.2$ & $14.8 \pm 2.7$ & $900.3 \pm 134.3$ & $15.3 \pm 5.0$ & $6.0 \pm 3.5$ & $8.2 \pm 2.6$ \\
\hline Other & - & - & - & - & $0.1 \pm 0.2$ & $0.2 \pm 0.2$ & - & - & - & - & $8.7 \pm 2.3$ \\
\hline$p$ value & $<0.001$ & 0.001 & $<0.001$ & 0.043 & $<0.001$ & $<0.001$ & $<0.001$ & $<0.001$ & $<0.001$ & $<0.001$ & 0.261 \\
\hline \multicolumn{12}{|l|}{$\begin{array}{l}\text { Graduate } \\
\text { status }\end{array}$} \\
\hline $\begin{array}{l}\text { Undergraduate } \\
\text { student }\end{array}$ & $1.6 \pm 0.6$ & $0.7 \pm 0.3$ & $1.3 \pm 0.3$ & $0.9 \pm 0.4$ & $0.3 \pm 0.3$ & $0.2 \pm 0.2$ & $15.3 \pm 3.1$ & $964.7 \pm 211.8$ & $16.4 \pm 6.2$ & $6.7 \pm 4.2$ & $8.3 \pm 2.7$ \\
\hline $\begin{array}{l}\text { Graduate } \\
\text { student }\end{array}$ & $1.6 \pm 0.6$ & $0.7 \pm 0.3$ & $1.4 \pm 0.4$ & $1.0 \pm 0.4$ & $0.3 \pm 0.3$ & $0.2 \pm 0.2$ & $16.4 \pm 3.3$ & $980.7 \pm 195.9$ & $16.0 \pm 5.4$ & $6.3 \pm 3.5$ & $7.7 \pm 2.7$ \\
\hline \multirow{2}{*}{\multicolumn{12}{|c|}{$\begin{array}{l}\text { Residency } \\
\text { status }\end{array}$}} \\
\hline & & & & & & & & & & & \\
\hline $\begin{array}{l}\text { Domestic } \\
\text { student }\end{array}$ & $1.6 \pm 0.6$ & $0.7 \pm 0.3$ & $1.3 \pm 0.3$ & $0.9 \pm 0.4$ & $0.3 \pm 0.3$ & $0.21 \pm 0.2$ & $15.5 \pm 3.1$ & $968.4 \pm 206.8$ & $16.4 \pm 6.1$ & $6.6 \pm 4.0$ & $8.3 \pm 2.6$ \\
\hline $\begin{array}{l}\text { International } \\
\text { student }\end{array}$ & $1.6 \pm 0.6$ & $0.7 \pm 0.3$ & $1.4 \pm 0.4$ & $1.0 \pm 0.4$ & $0.4 \pm 0.4$ & $0.15 \pm 0.2$ & $16.1 \pm 3.1$ & $917.4 \pm 215.8$ & $16.0 \pm 6.3$ & $6.8 \pm 4.7$ & $7.3 \pm 3.1$ \\
\hline$p$ value & 0.734 & 0.950 & 0.001 & 0.201 & 0.005 & 0.001 & 0.019 & 0.866 & 0.448 & 0.527 & $<0.001$ \\
\hline
\end{tabular}

Pearson correlation coefficients for relationships observed between dietary intakes, measured by the DSQ, and demographic measures. $\mathrm{M}=$ mean, $\mathrm{SD}=$ standard deviation. Missing data: gender $(\mathrm{N}=0)$; graduate status $(\mathrm{N}=0)$; residency status $(\mathrm{N}=0)$; dairy $(\mathrm{N}=41)$; whole grains $(\mathrm{N}=37)$; vegetables $(\mathrm{N}=37)$; fruits $(\mathrm{N}=37)$; red meat $(\mathrm{N}=4)$; processed meat $(\mathrm{N}=1276)$; fiber $(\mathrm{N}=37)$; calcium $(\mathrm{N}=37)$; total added sugar $(\mathrm{N}=40)$; total added sugar from SSB $(\mathrm{N}=52)$; dietary behaviors $(\mathrm{N}=0)$. Note that intake data could not be calculated for a gender other than male and female using the DSQ; therefore, intake data were missing for students who self-identified as "other". 


\subsection{Unadjusted and Adjusted Findings}

The results of the unadjusted models indicated that higher financial stress was associated with lower vegetable, fruit, fiber, and calcium intake; higher added sugar intake from SSB; and higher dietary risk scores (Table 5). When adjusted for age, gender, and residency status, the results showed that higher financial stress was no longer associated with calcium intake, but the other significant relationships in the unadjusted model remained. After the adjustment for perceived stress in addition to age, gender and residency status, the association between financial stress and dietary risk score was no longer significant, but all other relationships remained.

Table 5. Financial stress and its association with dietary intake and dietary risk score.

\begin{tabular}{|c|c|c|c|c|c|c|c|c|c|}
\hline \multirow[b]{2}{*}{ Financial Stress } & \multicolumn{3}{|c|}{ Model A } & \multicolumn{3}{|c|}{ Model B } & \multicolumn{3}{|c|}{ Model C } \\
\hline & B $(95 \%$ CI) & SE B & $p$ Value & $\mathrm{aB}(95 \% \mathrm{CI})$ & $\mathrm{SE}$ aB & $p$ Value & $\mathrm{aB}(95 \% \mathrm{CI})$ & $\mathrm{SE}$ aB & $p$ Value \\
\hline $\begin{array}{l}\text { Dairy intake } \\
\text { (cups/d) }\end{array}$ & $\begin{array}{c}-0.001 \\
(-0.006,0.005)\end{array}$ & 0.003 & 0.842 & $\begin{array}{c}0.002 \\
(-0.003,0.007)\end{array}$ & 0.002 & 0.392 & $\begin{array}{c}0.001 \\
(-0.004,0.007)\end{array}$ & 0.003 & 0.616 \\
\hline $\begin{array}{l}\text { Whole grains intake } \\
\text { (cups/d) }\end{array}$ & $\begin{array}{c}-0.003 \\
(-0.006,-0.0002)\end{array}$ & 0.002 & 0.072 & $\begin{array}{c}-0.003 \\
(-0.006,-0.0001)\end{array}$ & 0.002 & 0.047 & $\begin{array}{c}-0.002 \\
(-0.006,0.001)\end{array}$ & 0.002 & 0.146 \\
\hline $\begin{array}{l}\text { Vegetable intake } \\
(\text { cups } / d)\end{array}$ & $\begin{array}{c}-0.008 \\
(-0.011,-0.005)\end{array}$ & 0.002 & $<0.001$ & $\begin{array}{c}-0.007 \\
(-0.010,-0.004)\end{array}$ & 0.002 & $<0.001$ & $\begin{array}{l}-0.006 \\
(-0.009 \\
-0.003)\end{array}$ & 0.002 & $<0.001$ \\
\hline $\begin{array}{l}\text { Fruit intake } \\
\text { (cups/d) }\end{array}$ & $\begin{array}{c}-0.007 \\
(-0.011,-0.003)\end{array}$ & 0.002 & $<0.001$ & $\begin{array}{c}-0.007 \\
(-0.011,-0.003)\end{array}$ & 0.002 & 0.001 & $\begin{array}{l}-0.005 \\
(-0.009 \\
-0.001)\end{array}$ & 0.002 & 0.026 \\
\hline $\begin{array}{l}\text { Red meat intake } \\
\quad(\text { times } / d)\end{array}$ & $\begin{array}{c}-0.001 \\
(-0.004,0.002)\end{array}$ & 0.001 & 0.442 & $\begin{array}{c}0.0004 \\
(-0.002,0.003)\end{array}$ & 0.001 & 0.766 & $\begin{array}{c}0.002 \\
(-0.001,0.005)\end{array}$ & 0.002 & 0.317 \\
\hline $\begin{array}{l}\text { Processed meat } \\
\text { intake (times } / \mathrm{d} \text { ) }\end{array}$ & $\begin{array}{c}-0.00001 \\
(-0.002,0.002)\end{array}$ & 0.001 & 0.992 & $\begin{array}{c}0.0003 \\
(-0.002,0.002)\end{array}$ & 0.001 & 0.816 & $\begin{array}{c}-0.0005 \\
(-0.003,0.002)\end{array}$ & 0.001 & 0.667 \\
\hline Fiber intake (g/d) & $\begin{array}{c}-0.091 \\
(-0.121,-0.062)\end{array}$ & 0.015 & $<0.001$ & $\begin{array}{c}-0.082 \\
(-0.109,-0.055)\end{array}$ & 0.014 & $<0.001$ & $\begin{array}{c}-0.071 \\
(-0.099 \\
-0.042)\end{array}$ & 0.015 & $<0.001$ \\
\hline $\begin{array}{l}\text { Calcium intake } \\
(\mathrm{mg} / \mathrm{d})\end{array}$ & $\begin{array}{c}-2.153 \\
(-4.032,-0.274)\end{array}$ & 0.958 & 0.025 & $\begin{array}{c}-0.771 \\
(-2.334,0.793)\end{array}$ & 0.797 & 0.334 & $\begin{array}{c}-0.896 \\
(-2.551,0.759)\end{array}$ & 0.844 & 0.288 \\
\hline $\begin{array}{l}\text { Total added sugar } \\
\text { intake (tsp/d) }\end{array}$ & $\begin{array}{c}0.023 \\
(-0.030,0.076)\end{array}$ & 0.027 & 0.395 & $\begin{array}{c}0.038 \\
(-0.013,0.089)\end{array}$ & 0.026 & 0.140 & $\begin{array}{c}0.009 \\
(-0.045,0.063)\end{array}$ & 0.027 & 0.746 \\
\hline $\begin{array}{l}\text { Added sugar from } \\
\text { SSB intake (tsp/d) }\end{array}$ & $\begin{array}{c}0.059 \\
(0.021,0.097)\end{array}$ & 0.019 & 0.002 & $\begin{array}{c}0.068 \\
(0.030,0.106)\end{array}$ & 0.019 & $<0.001$ & $\begin{array}{c}0.054 \\
(0.014,0.094)\end{array}$ & 0.020 & 0.008 \\
\hline $\begin{array}{l}\text { Overall dietary } \\
\text { risk score }\end{array}$ & $\begin{array}{c}0.045 \\
(0.019,0.071)\end{array}$ & 0.013 & 0.001 & $\begin{array}{c}0.042 \\
(0.016,0.068)\end{array}$ & 0.013 & 0.002 & $\begin{array}{c}0.019 \\
(-0.008,0.046)\end{array}$ & 0.014 & 0.177 \\
\hline
\end{tabular}

Financial stress, as measured by USFSA scores, and the relationship between dietary intake of food groups and nutrients, measured by DSQ, and dietary risk score as measured by STC. B = beta coefficient; $95 \% \mathrm{CI}=95 \%$ confidence intervals; $\mathrm{SE}=$ standard error; $\mathrm{Ab}=$ adjusted beta coefficient. Model A: unadjusted. Model B: Model A + age, gender, residency status. Model C: Model B + perceived stress.

\subsection{Mediation Analysis}

The mediation analysis of model 1 , which examined poor sleepers, showed that financial stress and dietary risk score were not directly correlated, but they were indirectly correlated through poor sleep quality. This finding indicates that poor sleep quality completely mediated the relationship between financial stress and dietary risk score among students with poor sleep quality (Table 6). Additionally, higher financial stress was associated with poorer sleep quality, and poorer sleep quality was associated with a higher dietary risk score. However, the mediation effect of sleep quality on the relationship between financial stress and dietary risk score among students who reported good sleep quality was not significant (Table 7). Further, financial stress was not associated with sleep quality among students who reported good sleep quality. 
Table 6. Model 1 Mediation Analysis among Students with Poor Sleep Quality.

\begin{tabular}{ccccc}
\hline Variables & B & SE & t & $p$ Value \\
\hline Financial stress $\rightarrow$ poor sleep quality & 0.16 & 0.02 & 10.05 & $<0.001$ \\
Poor sleep quality $\rightarrow$ dietary risk score & 0.07 & 0.03 & 2.48 & 0.013 \\
Financial stress $\rightarrow$ dietary risk score & 0.02 & 0.02 & 1.12 & 0.262 \\
\hline Bootstrap & Effect & SE & LL 95\%CI & UL 95\%CI \\
\hline Poor sleep quality & 0.012 & 0.005 & 0.002 & 0.022 \\
\hline
\end{tabular}

Note: $\mathrm{N}=847$ in the model.

Table 7. Model 3 Mediation Analysis among Students with Good Sleep Quality.

\begin{tabular}{ccccc}
\hline Variables & B & SE & t & $p$ Value \\
\hline Financial stress $\rightarrow$ good sleep quality & -0.01 & 0.01 & -0.52 & 0.603 \\
Good sleep quality $\rightarrow$ dietary risk score & 0.23 & 0.11 & 2.16 & 0.031 \\
Financial stress $\rightarrow$ dietary risk score & 0.04 & 0.02 & LL 95\%CI & UL 95\%CI \\
\hline Bootstrap & Effect & SE & -0.009 & 0.004 \\
\hline Poor sleep quality & -0.001 & 0.003 & & 062 \\
\hline
\end{tabular}

Note: $\mathrm{N}=411$ in the model.

The mediation analysis of model 2 , which analyzed only short sleepers $(<7 \mathrm{~h}$ per night), showed that financial stress and dietary risk score were not directly correlated, but they were indirectly correlated through short sleep duration. This result indicates that short sleep duration completely mediated the relationship between financial stress and overall dietary risk score among students who did not meet sleep recommendations (Table 8). Additionally, higher financial stress was associated with shorter sleep duration, and shorter sleep duration was associated with higher dietary risk scores. However, the mediation effect of sleep quality on the relationship between financial stress and dietary risk score among students who reported meeting minimum sleep recommendations was not significant (Table 9). Finally, financial stress was not associated with sleep duration among students who reported adequate sleep duration.

Table 8. Model 2 Mediation Analysis of Students Not Meeting the Minimum Sleep Duration.

\begin{tabular}{ccccc}
\hline Variables & B & SE & t & $p$ Value \\
\hline Financial stress $\rightarrow$ short sleep duration & -0.02 & 0.01 & -2.04 & 0.043 \\
Short sleep duration $\rightarrow$ dietary risk score & -0.69 & 0.24 & 2.85 & 0.005 \\
Financial stress $\rightarrow$ dietary risk score & 0.03 & 0.03 & 0.78 & 0.436 \\
\hline Bootstrap & Effect & SE & LL 95\%CI & UL 95\%CI \\
\hline Short sleep duration & -0.01 & 0.007 & -0.026 & -0.0003 \\
\hline
\end{tabular}

Note: $\mathrm{N}=303$ in the model.

Table 9. Model 4 Mediation Analysis of Students Meeting the Minimum Sleep Duration.

\begin{tabular}{ccccc}
\hline Variables & B & SE & t & $p$ Value \\
\hline Financial stress $\rightarrow$ adequate sleep duration & -0.01 & 0.01 & -1.10 & 0.270 \\
Adequate sleep duration $\rightarrow$ dietary risk score & 0.19 & 0.09 & 2.05 & 0.041 \\
Financial stress $\rightarrow$ dietary risk score & 0.02 & 0.02 & 1.45 & 0.147 \\
\hline Bootstrap & Effect & SE & LL 95\%CI & UL 95\%CI \\
\hline Short sleep duration & -0.001 & 0.001 & -0.0043 & 0.0011 \\
\hline
\end{tabular}


Overall, the mediation analyses demonstrated that the relationship between financial stress and dietary risk score was completely mediated by sleep quality among students who reported poor sleep quality and completely mediated by sleep duration among students who reported sleeping $<7 \mathrm{~h}$ per night. This result indicates that the relationship between financial stress and overall dietary risk score was associated with poor sleep quality among poor quality sleepers and short sleep duration among short sleepers. Sleep quality and duration did not mediate the relationships between financial stress and dietary risk score for students reporting adequate or good quality sleep.

\section{Discussion}

The purpose of this study was to explore relationships between financial stress, dietary intake and behavior, and if sleep quality and duration mediated these relationships. As hypothesized, higher financial stress among higher education students was associated with lower vegetable, fruit, fiber, and calcium intake, higher added sugar intake from SSB, and higher dietary risk score. Further, the positive relationship between financial stress and dietary risk score was associated with both poor sleep quality among students who reported poor sleep quality and short sleep duration among students who did not meet the recommendation of at least $7 \mathrm{~h}$ of sleep per night. These results suggest that reducing financial stress and improving sleep quality and duration among higher education students could be effective strategies for improving dietary behaviors. These conclusions need further testing due to the cross-sectional nature of the present study.

Even though the relationships between financial stress and calcium intake and dietary risk scores disappeared after certain covariate adjustments, the lack of relationship after adjustment indicates that the covariates in question were significantly associated with these variables. For example, when adjusted for age, gender, and residency status, the results showed that higher financial stress was no longer associated with lower calcium intake. This finding indicates that these demographic covariates were associated with financial stress and calcium intake, but it should not be interpreted that there is no association between financial stress and calcium intake [54,55]. Additionally, after the adjustment of perceived stress in addition to age, gender, and residency status, the association between financial stress and dietary risk score was no longer significant. This indicates that perceived stress was associated with financial stress and dietary risk scores, but it should not be interpreted that no association between financial stress and dietary risk scores exists [54,55]. In summary, age, gender, and residency status were significant covariates in the relationship between financial stress and calcium intake while perceived stress was a significant covariate in the relationship between financial stress and dietary risk scores. Based on the bivariate correlations, gender was associated with calcium intake, and perceived stress was associated with both financial stress and dietary risk score. While the adjustment for covariates led to a loss of statistical significance, these findings do not indicate that there are no associations between financial stress and calcium intake or financial stress and dietary risk score.

\subsection{Associations between Financial Stress and Dietary Intake and Dietary Risk Score}

Stress, including financial stress, affects college students' eating habits, attitudes, and behaviors $[10,12,13,15,56,57]$. The present study found that higher financial stress among higher education students was associated with lower vegetable, fruit, fiber, and calcium intake; higher added sugar intake from SSB; and higher overall dietary risk score. The findings are consistent with previous studies showing higher financial stress is associated with more frequent unhealthy eating behaviors among higher education students [10,56,58-60]. Students tend to reduce their food purchasing budget when they experience economic hardship [12], and healthy foods, such as fruit and vegetables, are perceived as high-cost food items [15]. Further, lower fiber intake and calcium intake are associated with lower fruit and vegetable intake [61,62], which could further explain our findings regarding the association between higher financial stress and lower fiber 
and calcium intake. Given the evidence previously published and the current results, higher financial stress is associated with lower diet quality and more frequent engagement in risky dietary behaviors.

Contrary to our hypothesis, financial stress was not associated with lower dairy, higher red meat, processed meat, and total sugar intake. These findings suggest that factors other than financial concerns play a more important role in consumption of these items. Further study is needed to determine which other factors contribute to these consumption decisions.

\subsection{The Mediation Effect of Poor Sleep Quality on the Relationship between Financial Stress and Overall Dietary Risk Score}

As hypothesized, the present study found that poor sleep quality mediated the relationship between financial stress and dietary risk score among students who reported poor sleep quality. This finding indicates that greater financial stress may disrupt sleep by lowering sleep quality, and poor sleep quality is associated with more frequent engagement in undesirable dietary behaviors. Financial stress is a leading stressor among higher education students that contributes significantly to their overall perceived stress as well as their likelihood of persisting to degree completion [3-5]. Previous studies show that reducing perceived stress, which financial stress can contribute to, improved sleep quality $[63,64]$. Further, improving sleep quality led to improved eating behaviors $[65,66]$. Based on experimental studies noting the causal relationships between perceived stress and sleep quality as well as with sleep quality and dietary behaviors [63-66], the mediation effect of sleep quality on the relationship between financial stress and dietary risk score is plausible. Additionally, one other study explored the mediation effect of sleep quality on the relationship between food insecurity and obesity and revealed that poor sleep quality, specifically trouble falling asleep and maintaining sleep, mediated the relationship between food insecurity and obesity [67]. Because obesity is associated with undesirable dietary behaviors $[16,17]$, and based on the totality of the evidence, poor sleep quality appears to serve as an intermediate factor that explains the connection between financial stress and dietary risk score.

\subsection{The Mediation Effect of Short Sleep Duration on the Relationship between Financial Stress and Dietary Risk Score}

As hypothesized, the present study found that short sleep duration mediated the relationship between financial stress and dietary risk score among students reported short sleep duration. This finding is consistent with the finding from another study, in which it was noted that very short ( $\leq 4 \mathrm{~h}$ per night) and short sleep ( 5 to $6 \mathrm{~h}$ per night) duration mediated the relationship between food insecurity and obesity [67]. There are several mechanisms that could plausibly contribute to these relationships. First, stress, including financial stress, disrupts sleep and leads to short sleep duration through stress activates the hypothalamic-pituitary-adrenal (HPA) axis [68]. Stress hormone activation of the HPA axis leads to wakefulness and shortened sleep duration [69]. Second, sleep deprivation or curtailment has been associated with increased neural and behavioral reactivity to positive experiences [70]. For example, eating sweets is an enjoyable experience, and under the situation of sleep deprivation or curtailment, eating sweets becomes more enjoyable than usual, which could lead to overconsumption [71]. Based on the previous causal relationship studies, the previous mediation study, and the findings of the present study, short sleep duration could serve as an intermediate factor that explains why financial stress and overall dietary risk score are connected.

\subsection{Overall Public Health Messages}

Given the results of the present study, strategies for improving sleep quality and duration among higher education students may serve as a potential intervention to reduce risky dietary behaviors when financial stress is high. However, assessing sleep and providing sleep interventions have been overlooked among universities [72,73]. Despite the fact that 
higher education students have multiple demands on their time, various techniques have been successful in improving sleep outcomes in this population. These strategies include cognitive behavioral therapy for insomnia (CBTi), sleep hygiene education, mindfulness, relaxation, and hypnotherapy [74-78]. Further, one systematic review noted that CBTi was the most effective strategy for sleep improvement compared to other strategies [76], and its online delivery has also been shown to be effective [79]. Under situations like the current pandemic, online CBTi could be a feasible way to help students improve sleep. As both poor sleep quality and short sleep duration mediated the relationship between financial stress and dietary risk behaviors among poor sleepers, improving sleep when financial stress is high may prevent undesirable health behaviors or outcomes, like poor dietary choices or weight gain, among higher education students. Taken together, universities should consider feasible ways to improve the sleep of students in order to improve the health of students in higher education, especially when financial stress is a concern.

The present study suggests that financial stress reduction strategies could also serve as a potential intervention to reduce risky dietary behaviors among U.S. higher education students. Factors contributing to financial stress among higher education students include, not having enough money to participate in the same activities as peers, requiring additional finances to meet unexpected costs, sourcing additional finances from external loans, working more than $12 \mathrm{~h}$ per week, living costs, and expecting to have higher amounts of student loan debt at graduation [80,81]. Financial education and counseling are effective strategies to reduce financial stress and improve financial satisfaction among higher education students [82-86], and these strategies aim to help students identify sources and factors that cause financial stress as well as improve financial behaviors $[86,87]$. One metaanalysis noted that financial literacy is most effective when provided at the time of financial struggle [88]. Given the considerable evidence showing that the majority of the higher education students currently experience financial stress [9], addressing financial literacy at the university level could be an effective way to reduce financial stress and promote overall well-being of higher education students. Thus, university administrators should consider providing financial education and counseling to higher education students.

\subsection{Strengths and Limitations}

There are several strengths to this study. First, the large sample size in the present study provided adequate power to conduct the regression and mediation analyses. Second, using the mediation analysis allowed for the exploration of more complex relationships between financial stress, sleep duration and quality, and dietary risk score. Third, the study included students from three different large universities in the U.S., which strengthens our ability to generalize to students at other universities. Fourth, the study used validated tools for the higher education student population to measure financial stress, sleep, dietary intake, and dietary risk score $[35,45,89,90]$.

There are limitations to the study. First, the study is a cross-sectional study; therefore, it does not allow for the attribution of causality between the variables examined. Further investigations are needed to confirm possible causal relationships. Second, the study was not able to compare the combined mediation effects of poor sleep quality and short sleep duration on the relationships between financial stress and the dietary risk score because sleep quality and duration were measured using different scales and were assessed in two separate models. Standardized path analyses might be considered in the future to overcome this limitation. Third, the universities studied are large, public universities, and in-state tuition is relatively low compared to private institutions; although, there was no stipulation that students completing the surveys had to be in-state students. Fourth, the study did not collect socioeconomic status of the participants, which could be a possible covariate. Fifth, the study was conducted at the beginning of the COVID-19 pandemic, and studies demonstrate that the pandemic has influenced variables that we measured, including increased financial and perceived stress and worsened sleep [91,92]. In the present study, the majority of students indicated increased financial stress as a part of their pandemic experience. 
Therefore, these relationships should be examined again once financial stress, perceived stress, sleep, and life, in general, more closely resembles the pre-pandemic situation.

\section{Conclusions}

Sleep duration and quality were associated with poorer dietary quality based on intake data from multiple nutrients and food groups. For students who reported insufficient sleep or poor sleep quality, these sleep problems completely mediated the relationship between financial stress and dietary risk scores. To reduce the influence of financial stress on undesirable dietary behaviors, student programming should emphasize both sleep and financial literacy training.

Author Contributions: Conceptualization, C.D. and R.M.T.; methodology, C.D., W.W., P.Y.H., M.-J.L., R.M.T.; software, not applicable; validation, C.D.; formal analysis, C.D.; resources, R.M.T.; data curation, C.D., W.W., P.Y.H., M.-J.L. and R.M.T.; writing-original draft preparation, C.D., W.W., and R.M.T.; writing-review and editing, C.D., W.W., P.Y.H., M.-J.L. and R.M.T.; visualization, C.D.; supervision, R.M.T.; project administration, R.M.T.; funding acquisition, R.M.T. All authors have read and agreed to the published version of the manuscript.

Funding: This research was funded by the USDA National Institute of Food and Agriculture, Hatch \#1012976, and Michigan AgBioResearch and the Indiana University of Pennsylvania Faculty Incidental Research Support award.

Institutional Review Board Statement: The study was conducted according to the guidelines of the Declaration of Helsinki and approved by the Human Research Protection Program of Michigan State University (protocol code 00004285, approved 7 April 2020), the Institutional Review Board for the Protection of Human Subjects of the Indiana University of Pennsylvania (protocol code 20-101, approved 14 May 2020), and the Institutional Review Board of Bowling Green State University (protocol code 1599753; approved 29 April 2020).

Informed Consent Statement: Written informed consent has been obtained from the participants to publish this paper.

Data Availability Statement: The data presented in this study are available on request from the corresponding author. The data are not publicly available due to ongoing analyses.

Acknowledgments: The authors wish to thank graduate student Shengfang Song at Michigan State University for helping with preparing the DSQ data and undergraduate students Sara Yi Ling Folk, Suzannah Gadd, Kaylyn (Linyutong) Wang, and Xinyi Zhang at Michigan State University for helping with data preparation.

Conflicts of Interest: The funders had no role in the design of the study; in the collection, analyses, or interpretation of data; in the writing of the manuscript, or in the decision to publish the results.

\section{References}

1. Robb, C.A. College Student Financial Stress: Are the Kids Alright? J. Fam. Econ. Issues 2017, 38, 514-527. [CrossRef]

2. Zimmermann, M.; Bledsoe, C.; Papa, A. The Impact of the COVID-19 Pandemic on College Student Mental Health: A Longitudinal Examination of Risk and Protective Factors 2020. PsyArXiv 2020, preprint, 2-30. Available online: https://psyarxiv.com/ 2y7hu/ (accessed on 3 May 2021). [CrossRef]

3. Beiter, R.; Nash, R.; McCrady, M.; Rhoades, D.; Linscomb, M.; Clarahan, M.; Sammut, S. The prevalence and correlates of depression, anxiety, and stress in a sample of college students. J. Affect. Disord. 2015, 173, 90-96. [CrossRef] [PubMed]

4. Britt, S.L.; Ammerman, D.A.; Barrett, S.F.; Jones, S. Student Loans, Financial Stress, and College Student Retention. J. Stud. Financ. Aid 2017, 47. [CrossRef]

5. Xuereb, S. Why students consider terminating their studies and what convinces them to stay. Act. Learn. High. Educ. 2014, 15, 145-156. [CrossRef]

6. American College Health Association National College Health Assessment Spring 2019. Available online: https:/ /www.acha. org/documents/ncha/ncha-ii_spring_2019_us_reference_group_executive_summary.pdf (accessed on 13 April 2021).

7. Jones, H.E.; Manze, M.; Ngo, V.; Lamberson, P.; Freudenberg, N. The Impact of the COVID-19 Pandemic on College Students' Health and Financial Stability in New York City: Findings from a Population-Based Sample of City University of New York (CUNY) Students. J. Hered. 2021, 1-10. [CrossRef]

8. Hoyt, L.T.; Cohen, A.K.; Dull, B.; Castro, E.M.; Yazdani, N. “Constant Stress Has Become the New Normal”: Stress and Anxiety Inequalities among U.S. College Students in the Time of COVID-19. J. Adolesc. Health 2021, 68, 270-276. [CrossRef] 
9. Scholarship American. National College Student COVID-19 Survey Finds Financial Concerns Outpace Health Worries. Scholarsh. Am. 2020. Available online: https://scholarshipamerica.org/blog/national-college-student-covid-19-survey-findsfinancial-concerns-outpace-health-worries / (accessed on 13 April 2021).

10. Nelson, M.C.; Lust, K.; Story, M.; Ehlinger, E. Credit Card Debt, Stress and Key Health Risk Behaviors among College Students. Am. J. Health Promot. 2008, 22, 400-406. [CrossRef]

11. Hughes, R.; Serebryanikova, I.; Donaldson, K.; Leveritt, M. Student food insecurity: The skeleton in the university closet. Nutr. Diet. 2011, 68, 27-32. [CrossRef]

12. Grant-Smith, D.; De Zwaan, L. Don't spend, eat less, save more: Responses to the financial stress experienced by nursing students during unpaid clinical placements. Nurse Educ. Pr. 2019, 35, 1-6. [CrossRef]

13. Richardson, T.; Elliott, P.; Waller, G.; Bell, L. Longitudinal relationships between financial difficulties and eating attitudes in undergraduate students. Int. J. Eat. Disord. 2015, 48, 517-521. [CrossRef]

14. Hebden, L.; Chan, H.N.; Louie, J.C.; Rangan, A.; Allman-Farinelli, M. You Are What You Choose to Eat: Factors Influencing Young Adults' Food Selection Behaviour. Available online: https:/ / pubmed-ncbi-nlm-nih-gov.proxy2.cl.msu.edu/25891415/ (accessed on 5 November 2020).

15. Hartman, H.; Wadsworth, D.P.; Penny, S.; Van Assema, P.; Page, R. Psychosocial determinants of fruit and vegetable consumption among students in a New Zealand university. Results of focus group interviews. Appetite 2013, 65, 35-42. [CrossRef]

16. Lee, H.A.; Lee, W.K.; Kong, K.-A.; Chang, N.; Ha, E.-H.; Hong, Y.S.; Park, H. The Effect of Eating Behavior on Being Overweight or Obese During Preadolescence. J. Prev. Med. Public Health 2011, 44, 226-233. [CrossRef]

17. Kuźbicka, K.; Rachoń, D. Bad eating habits as the main cause of obesity among children. Pediatr. Endocrinol. Diabetes Metab. 2013, $19,106-110$.

18. La Vecchia, C.; DeCarli, A.; Pagano, R. Vegetable Consumption and Risk of Chronic Disease. Epidemiology 1998, 9, 208-210. [CrossRef]

19. Hariharan, D.; Vellanki, K.; Kramer, H. The Western Diet and Chronic Kidney Disease. Curr. Hypertens. Rep. 2015, 17, 1-9. [CrossRef]

20. Timlin, M.T.; Pereira, M.A. Breakfast Frequency and Quality in the Etiology of Adult Obesity and Chronic Diseases. Nutr. Rev. 2008, 65, 268-281. [CrossRef]

21. Michael, S.L.; Lowry, R.; Merlo, C.; Cooper, A.C.; Hyde, E.T.; McKeon, R. Physical activity, sedentary, and dietary behaviors associated with indicators of mental health and suicide risk. Prev. Med. Rep. 2020, 19, 101153. [CrossRef]

22. Ye, Y.-L.; Wang, P.-G.; Qu, G.-C.; Yuan, S.; Phongsavan, P.; He, Q.-Q. Associations between multiple health risk behaviors and mental health among Chinese college students. Psychol. Health Med. 2015, 21, 377-385. [CrossRef]

23. Keeter, S. Pew Research Center. Available online: https://www.pewresearch.org/fact-tank/2020/03/30/people-financiallyaffected-by-covid-19-outbreak-are-experiencing-more-psychological-distress-than-others/ (accessed on 13 April 2021).

24. Galambos, N.L.; Dalton, A.L.; Maggs, J.L. Losing Sleep over It: Daily Variation in Sleep Quantity and Quality in Canadian Students' First Semester of University. J. Res. Adolesc. 2009, 19, 741-761. [CrossRef]

25. Vgontzas, A.N.; Lin, H.-M.; Papaliaga, M.; Calhoun, S.; Vela-Bueno, A.; Chrousos, G.P.; Bixler, E.O. Short sleep duration and obesity: The role of emotional stress and sleep disturbances. Int. J. Obes. 2008, 32, 801-809. [CrossRef]

26. Klatt, M.D.; Buckworth, J.; Malarkey, W.B. Effects of Low-Dose Mindfulness-Based Stress Reduction (MBSR-ld) on Working Adults. Health Educ. Behav. 2008, 36, 601-614. [CrossRef]

27. Jones, B.J.; Kaur, S.; Miller, M.; Spencer, R.M.C. Mindfulness-Based Stress Reduction Benefits Psychological Well-Being, Sleep Quality, and Athletic Performance in Female Collegiate Rowers. Front. Psychol. 2020, 11, 11. [CrossRef]

28. Chaput, J.-P. Short Sleep Duration Promoting Overconsumption of Food: A Reward-Driven Eating Behavior? Sleep 2010, 33, 1135-1136. [CrossRef]

29. Brondel, L.; Romer, M.A.; Nougues, P.M.; Touyarou, P.; Davenne, D. Acute partial sleep deprivation increases food intake in healthy men. Am. J. Clin. Nutr. 2010, 91, 1550-1559. [CrossRef]

30. Spaeth, A.M.; Dinges, D.F.; Goel, N. Effects of Experimental Sleep Restriction on Weight Gain, Caloric Intake, and Meal Timing in Healthy Adults. Sleep 2013, 36, 981-990. [CrossRef]

31. Markwald, R.R.; Melanson, E.L.; Smith, M.R.; Higgins, J.; Perreault, L.; Eckel, R.H.; Wright, J.K.P. Impact of insufficient sleep on total daily energy expenditure, food intake, and weight gain. Proc. Natl. Acad. Sci. USA 2013, 110, 5695-5700. [CrossRef]

32. Peltz, J.S.; Bodenlos, J.S.; Kingery, J.N.; Rogge, R.D. The role of financial strain in college students' work hours, sleep, and mental health. J. Am. Coll. Health 2020, 1-8. [CrossRef]

33. Yang, C.-L.; Schnepp, J.; Tucker, R.M. Increased Hunger, Food Cravings, Food Reward, and Portion Size Selection after Sleep Curtailment in Women without Obesity. Nutrients 2019, 11, 663. [CrossRef]

34. Du, C.; Zan, M.; Cho, M.; Fenton, J.; Hsiao, P.; Hsiao, R.; Keaver, L.; Lai, C.-C.; Lee, H.; Ludy, M.-J.; et al. Health Behaviors of Higher Education Students from 7 Countries: Poorer Sleep Quality during the COVID-19 Pandemic Predicts Higher Dietary Risk. Clocks Sleep 2021, 3, 12-30. [CrossRef]

35. Lim, H.; Heckman, S.J.; Letkiewicz, J.C.; Montalto, C.P. Financial Stress, Self-Efficacy, and Financial Help-Seeking Behavior of College Students. J. Financ. Couns. Plan. 2014, 25, 148-160.

36. Cohen, S.; Kamarck, T.; Mermelstein, R. A global measure of perceived stress. J. Health Soc. Behav. 1983, 24, 385-396. [CrossRef] [PubMed] 
37. Buysse, D.J.; Hall, M.L.; Strollo, P.J.; Kamarck, T.W.; Owens, J.; Lee, L.; Reis, S.E.; Matthews, K.A. Relationships Between the Pittsburgh Sleep Quality Index (PSQI), Epworth Sleepiness Scale (ESS), and Clinical/Polysomnographic Measures in a Community Sample. J. Clin. Sleep Med. 2008, 4, 563-571. [CrossRef] [PubMed]

38. Guo, S.; Sun, W.; Liu, C.; Wu, S. Structural Validity of the Pittsburgh Sleep Quality Index in Chinese Undergraduate Students. Front. Psychol. 2016, 7, 1126. [CrossRef]

39. Beaudreau, S.A.; Spira, A.P.; Stewart, A.; Kezirian, E.J.; Lui, L.-Y.; Ensrud, K.; Redline, S.; Ancoli-Israel, S.; Stone, K.L. Validation of the Pittsburgh Sleep Quality Index and the Epworth Sleepiness Scale in older black and white women. Sleep Med. 2012, 13, 36-42. [CrossRef]

40. Marqués, D.; Gomes, A.A.; Meiavia, A.; Salgueiro, A.; Ribeiro, C.C.; Dischler, J. Reliability and initial validation of the Pittsburgh Sleep Quality Index, European Portuguese version: A preliminary study in a sample of higher education students. Sleep Med. 2013, 14, e140. [CrossRef]

41. Buysse, D.J.; Reynolds, C.F.; Monk, T.H.; Berman, S.R.; Kupfer, D.J. The Pittsburgh sleep quality index: A new instrument for psychiatric practice and research. Psychiatry Res. 1989, 28, 193-213. [CrossRef]

42. Hirshkowitz, M.; Whiton, K.; Albert, S.M.; Alessi, C.; Bruni, O.; DonCarlos, L.; Hazen, N.; Herman, J.; Katz, E.S.; Kheirandish-Gozal, L.; et al. National Sleep Foundation's sleep time duration recommendations: Methodology and results summary. Sleep Health 2015, 1, 40-43. [CrossRef]

43. Dietary Screener Questionnaires (DSQ) in the NHANES 2009-10: DSQ I EGRP/DCCPS/NCI/NIH. Available online: https: / / epi.grants.cancer.gov/nhanes/dietscreen/questionnaires.html (accessed on 12 April 2021).

44. Dietary Screener Questionnaire (DSQ) in the NHANES 2009-10: Data Processing \& Scoring Procedures | EGRP/DCCPS/NCI/NIH. Available online: https:/ / epi.grants.cancer.gov/nhanes/dietscreen/scoring/ (accessed on 12 April 2021).

45. Paxton, A.E.; Strycker, L.A.; Toobert, D.J.; Ammerman, A.S.; Glasgow, R.E. Starting the Conversation: Performance of a Brief Dietary Assessment and Intervention Tool for Health Professionals. Am. J. Prev. Med. 2011, 40, 67-71. [CrossRef]

46. Craig, C.L.; Marshall, A.L.; Sjöström, M.; Bauman, A.E.; Booth, M.L.; Ainsworth, B.E.; Pratt, M.; Ekelund, U.; Yngve, A.; Sallis, J.F.; et al. International Physical Activity Questionnaire: 12-Country Reliability and Validity. Med. Sci. Sports Exerc. 2003, 35, 1381-1395. [CrossRef]

47. Guidelines for Data Processing and Analysis of the International Physical Activity Questionnaire (IPAQ)-Short and Long Forms Contents. Available online: https: / / www.researchgate.net / file.PostFileLoader.html?id=5641f4c36143250eac8b45b7\&assetKey= AS\%3A294237418606593\%401447163075131 (accessed on 13 April 2021).

48. Thissen, D.; Steinberg, L.; Kuang, D. Quick and Easy Implementation of the Benjamini-Hochberg Procedure for Controlling the False Positive Rate in Multiple Comparisons. J. Educ. Behav. Stat. 2002, 27, 77-83. [CrossRef]

49. Lee, P.H.; Burstyn, I. Identification of confounder in epidemiologic data contaminated by measurement error in covariates. BMC Med. Res. Methodol. 2016, 16, 1-18. [CrossRef]

50. Bollen, K.A.; Bauldry, S. Three Cs in measurement models: Causal indicators, composite indicators, and covariates. Psychol. Methods 2011, 16, 265-284. [CrossRef]

51. Wolf, E.J.; Harrington, K.M.; Clark, S.L.; Miller, M.W. Sample Size Requirements for Structural Equation Models. Educ. Psychol. Meas. 2013, 73, 913-934. [CrossRef]

52. Hayes, A.F. Introduction to Mediation, Moderation, and Conditional Process Analysis, 2nd ed.; A Regression-Based Ap-Proach; Guilford Publications: New York, NY, USA, 2017; ISBN 978-1-4625-3465-4.

53. PROCESS: A Versatile Computational Tool for Observed Variable Mediation, Moderation, and Conditional Process Mod-eling 1. 2012. Available online: https:/ / www.semanticscholar.org/paper/PROCESS-\%3A-A-Versatile-Computational-Tool-for-\%2C\%2C-1-Hayes /862dd61cccad6b39ffe890557db19a6356bbe8ca (accessed on 12 April 2021).

54. MacKinnon, D.P.; Krull, J.L.; Lockwood, C.M. Equivalence of the Mediation, Confounding and Suppression Effect. Prev. Sci. 2000, 1, 173-181. [CrossRef]

55. Bias, Confounding and Effect Modification ISTAT 507. Available online: https://online.stat.psu.edu/stat507/lesson/3/3.5 (accessed on 27 April 2021).

56. Errisuriz, V.L.; Pasch, K.E.; Perry, C.L. Perceived stress and dietary choices: The moderating role of stress management. Eat. Behav. 2016, 22, 211-216. [CrossRef] [PubMed]

57. Debeuf, T.; Verbeken, S.; Van Beveren, M.-L.; Michels, N.; Braet, C. Stress and Eating Behavior: A Daily Diary Study in Youngsters. Front. Psychol. 2018, 9, 2657. [CrossRef]

58. Lee, A.; Yu, D.; Whang, J.; Yu, Q.; Yang, Z. HBI Institutional Responsibility: Sugar-Sweetened Beverages: Factors That Influence Motivation and Consumption, Correlates, and Interventions among University Students. Available online: https:/ /open.library. ubc.ca/cIRcle/collections/undergraduateresearch/18861/items/1.0375710 (accessed on 13 April 2021).

59. Elshurbjy, A.J.; Ellulu, M.S. Association between stress and dietary behaviors among university students: Mini-review. Med Clin. Arch. 2017, 1. [CrossRef]

60. Larson, N.; Laska, M.N.; Neumark-Sztainer, D. Food Insecurity, Diet Quality, Home Food Availability, and Health Risk Behaviors Among Emerging Adults: Findings From the EAT 2010-2018 Study. Am. J. Public Health 2020, 110, 1422-1428. [CrossRef]

61. Fleming, K.H.; Heimbach, J.T. Consumption of Calcium in the U.S.: Food Sources and Intake Levels. J. Nutr. 1994, 124, 1426S-1430S. [CrossRef] [PubMed] 
62. Bes-Rastrollo, M.; Martínez-González, M.Á.; Sánchez-Villegas, A.; Arrillaga, C.D.L.F.; Martínez, J.A. Association of fiber intake and fruit/vegetable consumption with weight gain in a Mediterranean population. Nutrients 2006, 22, 504-511. [CrossRef] [PubMed]

63. Klatt, M.; Norre, C.; Reader, B.; Yodice, L.; White, S. Mindfulness in Motion: A Mindfulness-Based Intervention to Reduce Stress and Enhance Quality of Sleep in Scandinavian Employees. Mindfulness 2017, 8, 481-488. [CrossRef]

64. Eliasson, A.H.; Kashani, M.; Mayhew, M.; Ude, A.; Hoffman, J.; Vernalis, M. Reducing Perceived Stress Improves Sleep Quality: A Longitudinal Outcomes Study. Chest 2010, 138, 913A. [CrossRef]

65. Gwin, J.A.; Leidy, H.J. Breakfast Consumption Augments Appetite, Eating Behavior, and Exploratory Markers of Sleep Quality Compared with Skipping Breakfast in Healthy Young Adults. Curr. Dev. Nutr. 2018, 2, nzy074. [CrossRef]

66. Al Khatib, H.K.; Hall, W.L.; Creedon, A.; Ooi, E.; Masri, T.; McGowan, L.; Harding, S.V.; Darzi, J.; Pot, G.K. Sleep extension is a feasible lifestyle intervention in free-living adults who are habitually short sleepers: A potential strategy for decreasing intake of free sugars? A randomized controlled pilot study. Am. J. Clin. Nutr. 2018, 107, 43-53. [CrossRef]

67. Narcisse, M.; Long, C.R.; Felix, H.; Rowland, B.; Bursac, Z.; McElfish, P.A. The Mediating Role of Sleep Quality and Quantity in the Link between Food Insecurity and Obesity across Race and Ethnicity. Obesity 2018, 26, 1509-1518. [CrossRef]

68. Han, K.S.; Kim, L.; Shim, I. Stress and Sleep Disorder. Exp. Neurobiol. 2012, 21, 141-150. [CrossRef]

69. Nicolaides, N.C.; Vgontzas, A.N.; Kritikou, I.; Chrousos, G. HPA Axis and Sleep. 2000. Available online: https://www.ncbi.nlm. nih.gov/books/NBK279071/ (accessed on 13 April 2021).

70. Banks, S.; Dinges, D.F. Behavioral and Physiological Consequences of Sleep Restriction. J. Clin. Sleep Med. 2007, 3, 519-528. [CrossRef]

71. Simon, S.L.; Field, J.; Miller, L.E.; DiFrancesco, M.; Beebe, D.W. Sweet/Dessert Foods Are More Appealing to Adolescents after Sleep Restriction. PLoS ONE 2015, 10, e0115434. [CrossRef]

72. Prichard, J.R.; Hartmann, M.E. Follow-up to Hartmann \& Prichard: Should universities invest in promoting healthy sleep? A question of academic and economic significance. Sleep Health 2019, 5, 320-325. [CrossRef]

73. Center for Collegiate Mental Health (CCMH) 2017 Annual Report. Available online: https:/ sites.psu.edu/ccmh/files/2018/01/ 2017_CCMH_Report-1r3iri4.pdf (accessed on 13 April 2021).

74. Levenson, J.C.; Miller, E.; Hafer, B.L.; Reidell, M.F.; Buysse, D.J.; Franzen, P.L. Pilot study of a sleep health promotion program for college students. Sleep Health 2016, 2, 167-174. [CrossRef]

75. Hershner, S.; O’Brien, L.M. The Impact of a Randomized Sleep Education Intervention for College Students. J. Clin. Sleep Med. 2018, 14, 337-347. [CrossRef]

76. Friedrich, A.; Schlarb, A.A. Let's talk about sleep: A systematic review of psychological interventions to improve sleep in college students. J. Sleep Res. 2017, 27, 4-22. [CrossRef]

77. Bowen, L.A. Assessing the effectiveness of online cognitive behavioral therapy in adults with chronic insomnia. J. Am. Acad. Physician Assist. 2020, 33, 1. [CrossRef]

78. Barber, L.K.; Cucalon, M.S. Modifying the Sleep Treatment Education Program for Students to include technology use (STEPSTECH): Intervention effects on objective and subjective sleep outcomes. Stress Health 2017, 33, 684-690. [CrossRef]

79. Peter, L.; Reindl, R.; Zauter, S.; Hillemacher, T.; Richter, K. Effectiveness of an Online CBT-I Intervention and a Face-to-Face Treatment for Shift Work Sleep Disorder: A Comparison of Sleep Diary Data. Int. J. Environ. Res. Public Health 2019, $16,3081$. [CrossRef]

80. Heckman, S.; Lim, H.; Montalto, C. Factors Related to Financial Stress among College Students. J. Financial Ther. $2014,5,3$. [CrossRef]

81. Gregory, K.; Matthew, S.; Baguley, J. Analysis of the costs of veterinary education and factors associated with financial stress among veterinary students in Australia. Aust. Veter J. 2018, 96, 11-16. [CrossRef]

82. Walstad, W.; Urban, C.; Asarta, C.J.; Breitbach, E.; Bosshardt, W.; Heath, J.; O’Neill, B.; Wagner, J.; Xiao, J.J. Perspectives on evaluation in financial education: Landscape, issues, and studies. J. Econ. Educ. 2017, 48, 93-112. [CrossRef]

83. Lyons, A.C. Credit Practices and Financial Education Needs of Midwest College Students. SSRN Electron. J. 2007. [CrossRef]

84. Lyons, A.C. Risky Credit Card Behavior of College Students. In Handbook of Consumer Finance Research; Springer: Berlin/Heidelberg, Germany, 2008; pp. 185-207. [CrossRef]

85. Gutter, M.S.; Copur, Z. Financial Behaviors and Financial Well-Being of College Students: Evidence from a National Survey. J. Fam. Econ. Issues 2011, 32, 699-714. [CrossRef]

86. Britt, S.L.; Canale, A.; Fernatt, F.; Stutz, K.; Tibbetts, R. Financial Stress and Financial Counseling: Helping College Students. J. Financial Couns. Plan. 2015, 26, 172-186. [CrossRef]

87. Britt, S.L.; Fernatt, F.; Nelson, J.S.; Yook, M.; Blue, J.M.; Canale, A.; Stutz, K.; Tibbetts, R. The Efficacy of Financial Counseling for College Students. Consum. Interests Annu. 2012, 58, 8.

88. Fernandes, D.; Lynch, J.G.; Netemeyer, R.G. Financial Literacy, Financial Education, and Downstream Financial Behaviors. Manag. Sci. 2014, 60, 1861-1883. [CrossRef]

89. Dietch, J.R.; Taylor, D.J.; Sethi, K.; Kelly, K.; Bramoweth, A.D.; Roane, B.M. Psychometric Evaluation of the PSQI in U.S. College Students. J. Clin. Sleep Med. 2016, 12, 1121-1129. [CrossRef]

90. Thompson, F.E.; Midthune, D.; Subar, A.F.; McNeel, T.; Berrigan, D.; Kipnis, V. Dietary intake estimates in the National Health Interview Survey, 2000: Methodology, results, and interpretation. J. Am. Diet. Assoc. 2005, 105, 352-363. [CrossRef] 
91. Martínez-Lezaun, I.; Santamaría-Vázquez, M.; Del Líbano, M. Influence of Confinement by COVID-19 on the Quality of Sleep and the Interests of University Students. Nat. Sci. Sleep 2020, 12, 1075-1081. [CrossRef] [PubMed]

92. Du, C.; Zan, M.C.H.; Cho, M.J.; Fenton, J.I.; Hsiao, P.Y.; Hsiao, R.; Keaver, L.; Lai, C.-C.; Lee, H.; Ludy, M.-J.; et al. Increased Resilience Weakens the Relationship between Perceived Stress and Anxiety on Sleep Quality: A Moderated Mediation Analysis of Higher Education Students from 7 Countries. Clocks Sleep 2020, 2, 334-353. [CrossRef] 